ISSN electrónico: 2445-1355

DOI: http://dx.doi.org/10.14201/fj2019426172

\title{
USO DE ANÁLOGOS DE SOMATOSTATINA EN LAS ANGIODISPLASIAS GASTROINTESTINALES REFRACTARIAS A OTROS TRATAMIENTOS
}

\section{Use of Somatostatin Analogues in Gastrointestinal Angiodysplasias Refractory to Other Treatments}

Gerardo VILLA DEL BOSQUE; Noemí ÁLVAREZ NÚÑEZ²; Ana Belén DOMÍNGUEZ CARBAJO²; Luis ORTEGA VALÍN $^{2}$

${ }^{1}$ Facultad de Farmacia de la Universidad de Salamanca.

2 Complejo Asistencial Universitario de León. Servicio de Farmacia Hospitalaria. Altos de Nava, s/n, 24071, León. Tel.: 987237400 (ext. 43355) Fax 98723882.

Correo-e: lortegav@saludcastillayleon.es

RESUMEN: La malformación vascular más común del tracto digestivo son las angiodisplasias gastrointestinales. Se caracterizan por una dilatación venocapilar con elevada fragilidad vascular, estado patológico que deriva en sangrados gastrointestinales. La prevalencia aumenta en pacientes de edad avanzada y pluripatológicos, lo que condiciona el tratamiento y pronóstico de las angiodisplasias.

El objetivo es evaluar la eficacia del tratamiento de las angiodisplasias con análogos de somatostatina, siendo estos el Octreótido y el Lanreótido (fuera de ficha técnica para la indicación), en condiciones de práctica clínica habitual. Además, se tratará de relacionar la respuesta terapéutica con la localización de las lesiones, comorbilidades, reingresos, transfusiones sanguíneas y prescripción de anticoagulantes.

Se llevó a cabo un estudio observacional retrospectivo con datos obtenidos de pacientes tratados en el Complejo Asistencial Universitario de León. Tras el análisis estadístico de los datos, se concluyó que no existen diferencias significativas entre los fármacos. Tampoco se encontró asociación significativa del éxito con las localizaciones de las angiodisplasias, ni con

Ediciones Universidad de Salamanca / @@ఠ $\quad$ FarmaJournal, vol. 4, núm. 2 (2019), pp. 61-72 
las comorbilidades coexistentes, ni con la toma de anticoagulantes. Solo tratamientos de larga duración (> 11 meses) se relacionan con indicadores objetivos de éxito, como la reducción del número de transfusiones y de reingresos.

Palabras clave: Octreótido; Lanreótido; Angiodisplasias gastrointestinales; Análogos de somatostatina.

ABSTRACT: The most common vascular malformation of the digestive tract are gastrointestinal angiodysplasias. They are characterized by a venocapillary dilation with high vascular fragility, a pathological state that results in gastrointestinal bleeding. The prevalence increases in elderly and multi-pathological patients, which conditions the treatment and prognosis of angiodysplasias.

The objective is to evaluate the efficacy of the treatment of angiodysplasias with somatostatin analogues, being these Octreotide and Lanreotide (outside of the technical data sheet for the indication), under conditions of usual clinical practice. In addition, we will try to relate the therapeutic response with the location of the lesions, comorbidities, readmissions, blood transfusions and prescription of anticoagulants.

A retrospective observational study was carried out with data obtained from patients treated at the Complejo Asistencial Universitario de León. After the statistical analysis of the data, it was concluded that there are no significant differences between the drugs. There was also no significant association of success with the locations of the angiodysplasias, nor with the coexisting comorbidities, nor with the taking of anticoagulants. Only long-term treatments (> 11 months) are related to objective indicators of success, such as reducing the number of transfusions and re-admissions.

Key words: Octreotide; Lanreotide; Gastrointestinal angiodisplasias; Somatostatin analogues.

\section{INTRODUCCIÓN}

La malformación vascular más común del tracto digestivo son las angiodisplasias gastrointestinales (GIADs) (Ramos-Rosario et al., 2016). Se caracterizan por una dilatación venocapilar (Arribas et al., 2017; Jackson et al., 2017), con elevada fragilidad vascular. Este estado patológico deriva en sangrados gastrointestinales (melenas, hematoquecia o hematemesis), cuya frecuencia será determinante para 
el ulterior desarrollo de una anemia ferropénica (Swanson et al., 2014). Presentan una prevalencia que aumenta en pacientes de edad avanzada (> 60 años) (Jackson et al., 2014) y pluripatológicos, siendo una condición que puede explicar la dificultad del tratamiento y pronóstico de las GIADs.

El tratamiento de elección es el endoscópico con plasma de argón (RamosRodario et al., 2016; Bon et al., 2012). El fracaso de la fulguración, la imposibilidad de acceso a la lesión, la extensa difusión de las GIADs a lo largo del tracto GI o la dificultad de sedación del paciente para realizar la enteroscopia, conllevarán la aplicación del tratamiento farmacológico (Molina et al., 2007; De la Peña et al., 2011).

La eficacia del arsenal terapéutico farmacológico no está demostrada con suficientes ensayos clínicos debido a la baja frecuencia de la enfermedad y la complejidad de constituir grupos de control (Molina et al., 2007).

En este estudio se analizará la eficacia de dos análogos de somatostatina en el tratamiento de GIADs (fuera de ficha técnica) en la práctica clínica real. Estos se caracterizan por su potente efecto vasoconstrictor esplácnico, la disminución del flujo portal y mesentérico, y su capacidad antiangiogénica (Molina et al., 2007; De La Peña et al., 2011). Son de aplicación tanto el Octreótido u Octreótido de larga duración como el Lanreótido.

\section{2. Овjetivos}

1. Evaluar la eficacia del tratamiento de GIADs con análogos de somatostatina en condiciones de práctica clínica habitual.

2. Como objetivo secundario se pretende relacionar la respuesta terapéutica con la localización de las lesiones, comorbilidades, reingresos, transfusiones sanguíneas y prescripción de anticoagulantes.

\section{Materiales y métodos}

Estudio observacional retrospectivo entre 2013 y 2017. Se incluyen pacientes adultos con sangrado gastrointestinal atribuible a GIADs refractarias al tratamiento endoscópico o quirúrgico.

\subsection{Criterios de inclusión}

1. Pacientes diagnosticados de GIADs mediante métodos endoscópicos (gastroscopia, colonoscopia o video cápsula endoscópica), tratados con análogos de somatostatina, y seguidos en el Hospital Universitario de León. 
G. VILLA DEL BOSQUE, N. ÁLVAREZ NÚÑEZ, A. B. DOMÍNGUEZ CARBAJO Y L. ORTEGA VALÍN USO DE ANÁLOGOS DE SOMATOSTATINA EN LAS ANGIODISPLASIAS GASTROINTESTINALES REFRACTARIAS A OTROS TRATAMIENTOS

2. Disponibilidad de pruebas diagnósticas, analíticas e informes clínicos.

3. Existencia de un periodo de seguimiento previo al tratamiento con análogos de somatostatina de duración similar al periodo de tratamiento.

4. Disponibilidad de la valoración médica subjetiva del paciente. Se emplea como criterio para la continuidad o suspensión del tratamiento.

\subsection{Criterios de exclusión}

1. Ausencia de datos de esenciales de seguimiento y valoración.

2. Fallecimiento del paciente sin llegar a iniciar el tratamiento.

3. Tratamiento previo con Talidomida.

4. Seguimiento del paciente fuera del Hospital Universitario de León.

\subsection{Fuentes de información}

1. Intranet del Hospital Universitario de León para la consulta de las historias clínicas.

2. Servicio de Transfusiones del Hospital de León.

3. Citrix, base de datos para consultar los registros de dispensación hospitalaria y la fecha de comienzo y fin de los tratamientos.

\subsection{Criterio de éxito}

Se consideró éxito terapéutico tanto la suspensión del tratamiento por el buen estado de salud del paciente (especificado en la historia clínica), como la continuación con el mismo fármaco (debido a que en caso de empeoramiento se procede a la sustitución inmediata del tratamiento). No se trata de un ensayo clínico en el que los periodos de tratamiento están preestablecidos, sino que es un estudio observacional basado en la práctica clínica real.

Debido a la gran heterogeneidad de los pacientes y sus tratamientos, solo se analizó la respuesta al primero de sus fármacos (Octreótido o Lanreótido), considerándose el cambio de un fármaco a otro como un fracaso terapéutico.

\subsection{Recogida y análisis de los datos}

1. Se creó una hoja de cálculo Excel 2010 (Microsoft Office ${ }^{\circledR}$ ) para recoger y tratar datos, y elaborar tablas y figuras. En la base de datos se recogió la edad, género, fármacos, duración del tratamiento, localización de las GIADs, comorbilidades, empleo de anticoagulantes y/o antiagregantes, y 
número de ingresos hospitalarios por servicio y de transfusiones sanguíneas a los 3, 6 y 11 meses previos y tras iniciar el tratamiento. Se utilizó la Calculadora epidemiológica del Servicio Vasco de Evaluación de Tecnologías Sanitarias para, mediante esta hoja de cálculo de Excel, estimar diferentes tipos de riesgo.

2. Mediante el programa SPSS ${ }^{\circledR}$-v.17 para Windows se realizó la estadística descriptiva (valores medios, desviación estándar y rango). El análisis de los fármacos y su éxito terapéutico se realizó con la prueba t de Student para muestras independientes, mientras que las diferencias entre periodos pre y post tratamiento con la t de Student para muestras relacionadas, con corrección de Tuckey e intervalos de confianza del $95 \%$. El análisis de asociación entre frecuencias de variables ordinales se realizó mediante Tablas de Contingencia empleando el test de Chi-Cuadrado, y el coeficiente de correlación no paramétrico de Spearman. Se utilizó la prueba $\mathrm{V}$ de Cramer para conocer el tamaño del efecto. Se considera $\mathrm{p}<0,05$ como nivel de significación.

Inicialmente se recogieron 55 pacientes tratados de GIADs con Octreótido o Lanreótido. Tras el análisis de los datos y la aplicación de los criterios establecidos, se excluyeron 15 ( 7 por traslado, 4 por fallecimiento, 1 por talidomida y 3 por falta de datos), incluyéndose 40 pacientes finalmente.

\section{Resultados}

Se incluyen en el estudio 40 pacientes, 25 hombres (62,5\%) y 15 mujeres $(37,5 \%)$. No se muestran diferencias entre los grupos de tratamiento y la edad $(82,5 \pm 6,1)$, la duración media del tratamiento (11 meses), la localización de las GIADs (aisladas o múltiples) y las comorbilidades asociadas (Tabla 1).

El $80 \%$ de los pacientes presentan GIADs en 2 o más localizaciones (múltiples). El porcentaje de localización de las GIADS no difiere entre ambos grupos de tratamiento, a excepción de las de colon que son el doble de frecuentes en grupo Octreótido. Las angiodisplasias aisladas son mayoritarias ( 43\%) en el grupo Lanreótido, y las de 3 ubicaciones en el grupo Octreótido ( 46\%).

En el $90 \%$ de los casos coexisten comorbilidades. Sus porcentajes tampoco difieren entre ambos grupos de tratamiento, a excepción de la insuficiencia cardiaca que se presenta significativamente con 3,5 veces mayor porcentaje en grupo Octreótido. 
G. VILLA DEL BOSQUE, N. ÁLVAREZ NÚÑEZ, A. B. DOMÍNGUEZ CARBAJO Y L. ORTEGA VALÍN USO DE ANÁLOGOS DE SOMATOSTATINA EN LAS ANGIODISPLASIAS GASTROINTESTINALES REFRACTARIAS A OTROS TRATAMIENTOS

Tabla 1. Características de la muestra tratada con Octreótido o Lanreótido, duración del tratamiento, localización de las angiodisplasias gastrointestinales (GIADs) y comorbilidades de los pacientes.

\begin{tabular}{|c|c|c|c|c|c|}
\hline & & $\begin{array}{l}\text { OCTREÓTIDO + } \\
\text { LANREÓTIDO } \\
(\mathrm{n}=40)(100 \%)\end{array}$ & $\begin{array}{l}\text { OCTREÓTIDO } \\
(\mathrm{n}=26)(65 \%)\end{array}$ & $\begin{array}{l}\text { LANREÓTIDO } \\
\qquad(\mathrm{n}=14)(35 \%)\end{array}$ & P-valor \\
\hline Edad (años) & & $82,5 \pm 6,1$ & $82,16 \pm 6,11$ & $83,07 \pm 6,28$ & 0,67 \\
\hline C'́n (o/) & Varón & $62,5 \%(n=25)$ & $69,2 \%(n=18)$ & $50 \%(n=7)$ & \\
\hline Genero (7o) & Mujer & $37,5 \%(n=15)$ & $30,8 \%(n=8)$ & $50 \%(n=7)$ & \\
\hline Duración trata & miento (meses) & $11 \pm 8,78$ & $12 \pm 8,75$ & $9,14 \pm 8,85$ & 0,333 \\
\hline Tipo de & Aislada (=1) & $20 \%(n=8)$ & & & \\
\hline localización & Múltiple ( $\geq 2)$ & $2,5 \pm 1,03$ & $2,81 \pm 0,84$ & $1,93 \pm 0,99$ & 0,009 \\
\hline Comprbilided & Total & $6,7 \pm 2,9$ & $6,5 \pm 3,21$ & $7,07 \pm 2,4$ & 0,564 \\
\hline Comor & Directa relación & $2,25 \pm 1,08$ & $2,1 \pm 1,07$ & $2,5 \pm 1,09$ & 0,289 \\
\hline LOCALIZACI & ÓN DE LAS AN & GIODISPLASIA & $\mathrm{S}(\%)$ & & \\
\hline - Ciego & & 37,5 & 46,2 & 21,4 & 0,13 \\
\hline - Duodeno & & 60 & 57,7 & 64,3 & 0,694 \\
\hline - Yeyuno & & 47,5 & 53,8 & 35,7 & 0,285 \\
\hline • Íleon & & 17,5 & 19,2 & 14,3 & 0,704 \\
\hline - Colon & & 42,5 & 53,8 & 21,4 & 0,049 \\
\hline - Gástricas & & 45 & 50 & 35,7 & 0,399 \\
\hline COMORBILII & ADES JUNTO & LAS ANGIOD & ISPLASIAS (\% & & \\
\hline - Insuficienc & ia Renal Crónica & 50 & 46,2 & 57,1 & 0,52 \\
\hline - Insuficienc & ia Cardiaca & 65 & 53,8 & 14,3 & 0,045 \\
\hline - Patología & Jalvular & 47,5 & 50 & 42,9 & 0,676 \\
\hline - Fibrilación & Auricular & 52,5 & 46,2 & 64,3 & 0,285 \\
\hline - Cirrosis & & 10 & 15,4 & 0 & 0,128 \\
\hline - Hiperten & sión Arterial & 87,5 & 80,8 & 100 & 0,83 \\
\hline - Diabetes & Mellitus & 40 & 34,6 & 50 & 0,356 \\
\hline - Hernia d & Hiato & 20 & 19,2 & 21,4 & 0,872 \\
\hline - Dislipem & & 52,5 & 46,2 & 64,3 & 0,285 \\
\hline - Hiperuri & cemia & 27,5 & 26,9 & 28,6 & 0,914 \\
\hline - Ninguna & & 10 & 7,7 & 7,1 & \\
\hline
\end{tabular}

Valores medios, desviación estándar y rango (valor mínimo-valor máximo). Donde: «p»= nivel de significación entre Octreótido y Lanreótido. $n=$ tamaño muestral. Comorbilidades directamente relacionadas: Insuficiencia Renal, Insuficiencia cardiaca, Patología valvular, Hipertensión arterial, Cirrosis. 
G. VILLA DEL BOSQUE, N. ÁLVAREZ NÚÑEZ, A. B. DOMÍNGUEZ CARBAJO Y L. ORTEGA VALÍN USO DE ANÁLOGOS DE SOMATOSTATINA EN LAS ANGIODISPLASIAS GASTROINTESTINALES REFRACTARIAS A OTROS TRATAMIENTOS

El éxito terapéutico no difiere significativamente en ambos grupos. Presentan un éxito medio del $50 \%$. La Tabla 2 muestra el porcentaje de contribución de los criterios utilizados a tal efecto (continuar con Lanreótido duplica en porcentaje al Octreótido), sin que presenten diferencias significativas.

TABLA 2. Porcentaje desagregado de éxito y fracaso en cada grupo de tratamiento.

\begin{tabular}{|c|c|c|c|c|c|c|c|}
\hline \multirow{2}{*}{\multicolumn{2}{|c|}{$\begin{array}{l}\text { EFICACIA DEL } 10^{\text {er }} \text { TRATAMIENTO }(n=40) \\
\text { Criterios: }\end{array}$}} & \multicolumn{2}{|c|}{ OCT + LAN } & \multicolumn{2}{|c|}{ OCT } & \multicolumn{2}{|c|}{ LAN } \\
\hline & & $\mathrm{n}$ & $\%$ & $\mathbf{n}$ & $\%$ & $\mathbf{n}$ & $\%$ \\
\hline \multirow{2}{*}{ Éxito $(50 \%)$ : } & Alta médica & 7 & 17,5 & 5 & 19,2 & 2 & 14,3 \\
\hline & Continúa Tratamiento & 13 & 32,5 & 6 & 23,1 & 7 & 50 \\
\hline \multirow{3}{*}{ Fracaso $(50 \%)$ : } & Cambiar Tratamiento & 7 & 17,5 & 4 & 15,4 & 3 & 21,4 \\
\hline & Muerte & 10 & 25 & 8 & 30,8 & 2 & 14,3 \\
\hline & Sin Tratamiento Análogo & 3 & 7,5 & 3 & 11,5 & 0 & 0 \\
\hline
\end{tabular}

Donde: \% = Porcentaje; $n=$ tamaño muestral; Oct = Octreótido; Lan = Lanreótido.

No se observan diferencias en la eficacia terapéutica con respecto al número de ingresos o la cantidad de transfusiones recibidas en los periodos de 3, 6 y 11 meses previos y posteriores al inicio del tratamiento, salvo en el número de trasfusiones a 3 meses con Octreótido, al reducirse significativamente a la mitad. No obstante, aumenta el porcentaje de pacientes que no presentan ningún reingreso, o no requieren ninguna transfusión en dichos periodos temporales, salvo los reingresos en grupo Lanreótido (Tabla-3).

TABLA 3. Criterios de eficacia con fármacos análogos de somatostatina en función del tiempo de tratamiento.

\begin{tabular}{|c|c|c|c|c|}
\hline \multicolumn{5}{|c|}{ 1. ${ }^{\text {er }}$ Criterio de eficacia: NÚMERO DE INGRESOS Y TRANSFUSIONES } \\
\hline & & $\begin{array}{l}\text { Periodo de } \\
\text { Tratamiento }\end{array}$ & $\begin{array}{c}\text { PRE- } \\
\text { Tratamiento }\end{array}$ & $\begin{array}{c}\text { POST- } \\
\text { Tratamiento }\end{array}$ \\
\hline \multirow{6}{*}{$\begin{array}{l}\text { OCTREÓTIDO + } \\
\text { LANREÓTIDO } \\
(\mathrm{n}=40)\end{array}$} & \multirow{3}{*}{ Ingresos $\left(n .^{\circ}\right)$} & 3 meses & $1,02 \pm 0,99$ & $0,85 \pm 0,89$ \\
\hline & & 6 meses & $1,35 \pm 1,4$ & $1,07 \pm 1,47$ \\
\hline & & 11 meses & $2,18 \pm 1,9$ & $2,24 \pm 2,34$ \\
\hline & \multirow{3}{*}{ Transfusión (n. $\left.{ }^{\circ}\right)$} & 3 meses & $5,92 \pm 4,57$ & $3,05 \pm 4,35 *$ \\
\hline & & 6 meses & $6,45 \pm 6,42$ & $4,67 \pm 7,69$ \\
\hline & & 11 meses & $10,16 \pm 8,41$ & $10,66 \pm 15,58$ \\
\hline
\end{tabular}


G. VILla DEL BOSQUE, N. ÁLVAREZ NÚÑEZ, A. B. DOMÍNGUEZ CARBAJO Y L. ORTEGA VALÍN USO DE ANÁLOGOS DE SOMATOSTATINA EN LAS ANGIODISPLASIAS GASTROINTESTINALES REFRACTARIAS A OTROS TRATAMIENTOS

\begin{tabular}{|c|c|c|c|c|}
\hline \multicolumn{5}{|c|}{ 1. ${ }^{\text {er }}$ Criterio de eficacia: NÚMERO DE INGRESOS Y TRANSFUSIONES } \\
\hline & & $\begin{array}{c}\text { Periodo de } \\
\text { Tratamiento }\end{array}$ & $\begin{array}{c}\text { PRE- } \\
\text { Tratamiento }\end{array}$ & $\begin{array}{c}\text { POST- } \\
\text { Tratamiento }\end{array}$ \\
\hline \multirow{6}{*}{$\begin{array}{l}\text { OCTREÓTIDO } \\
(\mathrm{n}=26)\end{array}$} & \multirow{3}{*}{ Ingresos $\left(\mathrm{n} .^{\circ}\right)$} & 3 meses & $1,04 \pm 0,82$ & $0,82 \pm 0,98$ \\
\hline & & 6 meses & $1,42 \pm 1,39$ & $1 \pm 1,38$ \\
\hline & & 11 meses & $2,4 \pm 1,91$ & $2,36 \pm 2,38$ \\
\hline & \multirow{3}{*}{ Transfusión (n. ${ }^{\circ}$ ) } & 3 meses & $5,88 \pm 4,55$ & $2,81 \pm 4,06 *$ \\
\hline & & 6 meses & $6,53 \pm 6,92$ & $4,65 \pm 7,66$ \\
\hline & & 11 meses & $10,6 \pm 8,39$ & $11,24 \pm 17,07$ \\
\hline \multirow{6}{*}{$\begin{array}{l}\text { LANREÓTIDO } \\
(n=14)\end{array}$} & \multirow{3}{*}{ Ingresos $\left(\mathrm{n} .^{\circ}\right)$} & 3 meses & $1 \pm 1,3$ & $0,93 \pm 0,73$ \\
\hline & & 6 meses & $1,21 \pm 1,48$ & $1,21 \pm 1,67$ \\
\hline & & 11 meses & $1,77 \pm 1,88$ & $2 \pm 2,35$ \\
\hline & \multirow{3}{*}{ Transfusión (n. $\left.{ }^{\circ}\right)$} & 3 meses & $6 \pm 4,77$ & $3,5 \pm 4,99$ \\
\hline & & 6 meses & $6,28 \pm 5,58$ & $4,71 \pm 8,04$ \\
\hline & & 11 meses & $10,21 \pm 6,6$ & $9,64 \pm 13,06$ \\
\hline \multicolumn{5}{|c|}{ 2. ${ }^{\circ}$ Criterio de eficacia: AUSENCIA DE REINGRESO O DE TRANSFUSION } \\
\hline & & $\begin{array}{l}\text { Periodo de } \\
\text { Tratamiento }\end{array}$ & $\begin{array}{c}\text { PRE- } \\
\text { Tratamiento } \\
(\%)\end{array}$ & $\begin{array}{c}\text { POST- } \\
\text { Tratamiento } \\
(\%)\end{array}$ \\
\hline \multirow{6}{*}{$\begin{array}{l}\text { OCTREÓTIDO + } \\
\text { LANREÓTIDO } \\
(\mathrm{n}=40)\end{array}$} & \multirow{3}{*}{$\begin{array}{l}\text { FRECUENCIA } \\
\text { DE NINGÚN } \\
\text { INGRESO }\end{array}$} & 3 meses & 30 & 40 \\
\hline & & 6 meses & 37,5 & 50 \\
\hline & & 11 meses & 12,5 & 20 \\
\hline & \multirow{3}{*}{$\begin{array}{l}\text { FRECUENCIA } \\
\text { DE NINGUNA } \\
\text { TRANSFUSIÓN }\end{array}$} & 3 meses & 15 & 52,5 \\
\hline & & 6 meses & 30 & 50 \\
\hline & & 11 meses & 7,5 & 22,5 \\
\hline \multirow{6}{*}{$\begin{array}{l}\text { OCTREÓTIDO } \\
(n=26)\end{array}$} & \multirow{3}{*}{$\begin{array}{l}\text { FRECUENCIA } \\
\text { DE NINGÚN } \\
\text { INGRESO }\end{array}$} & 3 meses & 26,9 & 50 \\
\hline & & 6 meses & 34,6 & 53,8 \\
\hline & & 11 meses & 7,7 & 23,1 \\
\hline & \multirow{3}{*}{$\begin{array}{l}\text { FRECUENCIA } \\
\text { DE NINGUNA } \\
\text { TRANSFUSIÓN }\end{array}$} & 3 meses & 19,2 & 53,8 \\
\hline & & 6 meses & 30,8 & 50 \\
\hline & & 11 meses & 0 & 15,4 \\
\hline \multirow{6}{*}{$\begin{array}{l}\text { LANREÓTIDO } \\
(n=14)\end{array}$} & \multirow{3}{*}{$\begin{array}{l}\text { FRECUENCIA } \\
\text { DE NINGÚN } \\
\text { INGRESO }\end{array}$} & 3 meses & 35,7 & 21,4 \\
\hline & & 6 meses & 42,9 & 42,9 \\
\hline & & 11 meses & 21,4 & 14,3 \\
\hline & \multirow{3}{*}{$\begin{array}{l}\text { FRECUENCIA } \\
\text { DE NINGUNA } \\
\text { TRANSFUSIÓN }\end{array}$} & 3 meses & 7,1 & 50 \\
\hline & & 6 meses & 28,6 & 50 \\
\hline & & 11 meses & 14,3 & 35,7 \\
\hline
\end{tabular}

Valores medios \pm desviación estándar. Donde: $n .{ }^{\circ}=$ número; $\%=$ porcentaje; $\mathrm{n}=$ tamaño muestra. Diferencias significativas entre Pre y Post-tratamiento: $* * \mathrm{P}<0,01$ 
G. VILLA DEL BOSQUE, N. ÁLVAREZ NÚÑEZ, A. B. DOMÍNGUEZ CARBAJO Y L. ORTEGA VALÍN USO DE ANÁLOGOS DE SOMATOSTATINA EN LAS ANGIODISPLASIAS GASTROINTESTINALES REFRACTARIAS A OTROS TRATAMIENTOS

El estudio de las tablas cruzadas de contingencia no mostró asociación (dependencia) entre el fármaco y el éxito terapéutico, ni con las localizaciones de las GIADs (salvo las de colon, con un tamaño del efecto moderado de 0,313), ni con las comorbilidades coexistentes (salvo la insuficiencia cardiaca, con un tamaño del efecto moderado de 0,319).

Tampoco hay asociación (dependencia) entre el éxito terapéutico con las localizaciones de las GIADs (salvo las de íleon, con un tamaño del efecto moderado de 0,334), ni con ninguna de las comorbilidades coexistentes.

Las correlaciones entre el porcentaje de éxito terapéutico farmacológico tanto con las localizaciones de las GIADs como con las comorbilidades coexistentes son muy bajas o inexistentes (salvo la moderada y significativa con el íleon (Correlación de Spearman =0,329)).

Ahora bien, el éxito terapéutico se correlaciona negativa y significativamente de forma moderada con el número de ingresos ( $\mathrm{r}=-0,336)$, y alta con el número de transfusiones $(r=-0,710)$ cuanto mayor sea el periodo temporal de tratamiento.

Finalmente, el tomar anticoagulantes (65 \% de la muestra) o no, tampoco mostró asociación (dependencia) ni correlación con el fármaco ni con el éxito terapéutico obtenido $(r=0,155)$.

\section{Discusión}

Tras el fracaso de la primera línea de tratamiento (cauterización de las GIADs con el tratamiento endoscópico de plasma de argón refractarios o no candidatos a tratamiento endoscópico o quirúrgico) se recurre al empleo fuera de ficha técnica del tratamiento farmacológico. En nuestro estudio, la segunda línea terapéutica fueron los análogos de somatostatina.

La selección de esta terapia no se estipuló con el objetivo de conseguir una homogeneidad de la muestra poblacional, sino que el criterio fue la práctica clínica médica habitual. Estudios como el de Roman-Rosario et al. (2016) muestran beneficios marginales en grupos muy seleccionados y reducidos (Brown et al., 2010), siendo poco representativos de este grupo de pacientes.

Tal y como presentamos en nuestros resultados, contamos con un tamaño muestral de 40 pacientes (elevado respecto a otros estudios como el de RamosRosario et at. (2016) o el de Molina et al. (2007)). No hemos demostrado diferencias en la eficacia terapéutica de un fármaco sobre otro, ni en su éxito terapéutico (57,7 \%-64,3\%), posiblemente condicionado por la fragilidad de los pacientes caracterizados por la edad avanzada ( $82,5 \pm 6,1$ años), el alto número de comorbilidades $(6,7 \pm 2,9)$ y la dispersión de las GIADs (80\% con localizaciones múltiples).

Respecto a la localización, Scaglione et al. (2007) refieren que todos los pacientes que respondieron tenían angiodisplasias en intestino delgado, solas o en 
combinación con el estómago o el colon. Nuestro trabajo no muestra asociación entre las diferentes localizaciones con el éxito terapéutico, salvo la localización de la GIADs en el íleon.

No obstante, en nuestros resultados observamos que clínicamente solo el Octreótido reduce significativamente las trasfusiones en los 3 primeros meses. Además, el análisis revela que una mayor duración del tratamiento ( 11 meses) se correlaciona con reducciones tanto en las transfusiones como en el número de reingresos, de forma similar a Molina et al. (2009).

Nuestros datos son coincidentes con el estudio de Bon et al. (2012), el cual presenta 12 pacientes tratados con Octreótido-LAR y 3 con Lanreótido, con una edad media de 76 años. Muestran eficacia significativa para reducir el número de transfusiones, favorecido por el amplio periodo de seguimiento ( 30 meses previos y 12 durante el tratamiento), similar al nuestro.

Las comorbilidades podrían incrementar la dificultad de tratamiento, según Molina et al. (2007), si bien nuestro estudio no lo ha puesto de manifiesto, pues solo se han relacionado los análogos de somatostatina con la insuficiencia cardíaca.

Nuestros resultados no muestran diferencias ni asociación ni correlación entre el empleo de anticoagulantes/antiagregantes con la eficacia del tratamiento. Esto se constata en los estudios de Ramos-Rosario et al. (2016) y Molina et al. (2007), los cuales obtienen resultados exitosos en el tratamiento a pesar del uso concomitante de anticoagulantes/antiagregantes (Blich et al., 2003).

La principal limitación de estos estudios es la pequeña muestra poblacional (debida a la baja prevalencia de la enfermedad) como también exponen RamosRosario et al. (2016), Molina et al. (2007) y Scaglione et al. (2007). Entendemos que ello se corrige en parte en nuestro estudio, si bien persiste la gran heterogeneidad de pacientes, con elevadas comorbilidades, múltiples localizaciones y edad avanzada (Molina et al., 2012). La otra gran limitación es, por razones éticas, la ausencia de grupo de control.

Sería interesante implementar el estudio analizando la respuesta de los casos en los que se sustituyó el Octreótido por el Lanreótido, o viceversa, para avalar o no la utilidad del intercambio terapéutico entre análogos de somatostatina, pudiendo mejorar así el estudio de Ramos-Rosario et al. (2016) en el que presentan 2 casos de GIADs refractarias a Octreótido-LAR tratadas con Lanreótido.

\section{Conclusiones}

- En nuestra muestra encontramos una alta mortalidad, posiblemente relacionada con una edad elevada y alta comorbilidad. Octreótido y Lanreótido muestran similares porcentajes de éxito terapéutico. No encontramos 
G. VILLA DEL BOSQUE, N. ÁLVAREZ NÚÑEZ, A. B. DOMÍNGUEZ CARBAJO Y L. ORTEGA VALÍN

USO DE ANÁLOGOS DE SOMATOSTATINA EN LAS ANGIODISPLASIAS

GASTROINTESTINALES REFRACTARIAS A OTROS TRATAMIENTOS

asociación significativa del éxito con las localizaciones de las GIADs, ni con las comorbilidades coexistentes, ni con la toma de anticoagulantes.

- Solo tratamientos de larga duración (> 11 meses) se relacionan con indicadores objetivos de éxito, como reducción del número de transfusiones y de reingresos. En este sentido, el Octreótido pudiera ser más eficaz a corto plazo en cuanto a reducir número de transfusiones.

- El valor de este estudio radica en el análisis del éxito terapéutico (50\% de los pacientes) en condiciones de práctica clínica real.

\section{Bibliografía}

Arribas Anta J, Zaera de la Fuente C, Martín Mateos R, González Martín J, Cañete Ruiz A, Boixeda de Miquel D. et al. Evaluación de la eficacia terapéutica endoscópica en hemorragia digestiva secundaria a angiodisplasias. Rev Gastroenterol Mex. 2017; 82(1):26-31.

Blich M, Fruchter O, Edelstein S, Edoute Y. Somatostatin therapy ameliorates chronic and refractory gastrointestinal bleeding caused by diffuse angiodysplasia in a patient on anticoagulation therapy. Scand J Gastroenterol. 2003; 38(7):801-803.

Bon C, Aparicio T, Vincent M, Mavros M, Bejou B, Raynaud JJ, et al. Long-acting somatostatin analogues decrease blood transfusion requirements in patients with refractory gastrointestinal bleeding associated with angiodysplasia. Aliment Pharmacol Ther. 2012; 36(6):587-593.

Brown C, Subramanian V, Mel Wilcox C, Peter S. Somatostatin Analogues in the Treatment of Recurrent Bleeding from Gastrointestinal Vascular Malformations: An Overview and Systematic Review of Prospective Observational Studies. Dig Dis Sci. 2010; 55(8):2129-2134.

De La Peña Trigueros M, Gutiérrez Macías A, Estrada Vicente R, Miguel de la Villa F. Utilidad del octreótido long-acting release en el sangrado digestivo de origen oscuro. Gac Med Bilbao. 2011; 108(2):57-59.

Jackson CS, Gerson LB. Management of Gastrointestinal Angiodysplastic Lesions (GIADs): A Systematic Review and Meta-Analysis. Am J Gastroenterol. 2014; 109(4):474-483.

Jackson CS, Strong R. Gastrointestinal Angiodysplasia. Diagnosis and Management. Gastrointest Endosc Clin N Am. 2017; 27(1):51-62.

Molina IJ, Pérez GB, Fernández Bermejo M. Avances en el tratamiento farmacológico de la hemorragia digestiva de origen oscuro. Rev Esp Enferm Dig. 2007; 99(8):457-462.

Molina IJ, Pérez GB, Hernández AM, Mateos Rodríguez J, Dueñas SC, Fernández Bermejo $\mathrm{M}$. Octreótido long acting release para la hemorragia digestiva en pacientes de edad avanzada con comorbilidad. Med Clin (Barc). 2009; 133(17):667-670.

Nardone G, Rocco A, Balzano T, Budillon G. The efficacy of octreotide therapy in chronic bleeding due to vascular abnormalities of the gastrointestinal tract. Aliment Pharmacol Ther. 1999; 13(11):1429-1436. 


\section{G. VILLA DEL BOSQUE, N. ÁLVAREZ NÚÑEZ, A. B. DOMÍNGUEZ CARBAJO Y L. ORTEGA VALÍN USO DE ANÁLOGOS DE SOMATOSTATINA EN LAS ANGIODISPLASIAS GASTROINTESTINALES REFRACTARIAS A OTROS TRATAMIENTOS}

Ramos-Rosario H, Badia Aranda E, Martín Lorente JL, Arias García L, Sicilia Aladrén B, Sáez-Royuela F. Eficacia de lanreótido en pacientes con angiodisplasias gastrointestinales refractarias al tratamiento con octreótido. Gastroenterol Hepatol. 2016; 39(3):213-214.

Sandoval Riveros C, Lúquez Mindiola A, Marulanda Fernández H, Otero Regino W. Sangrado del intestino delgado: enfoque y tratamiento. Rev Col Gastroenterol. 2017; 32(3):245-257.

Scaglione G, Pietrini L, Russo F, Franco MR, Sorrentini I. Long-acting octreotide as rescue therapy in chronic bleeding from gastrointestinal angiodysplasia. Aliment Pharmacol Ther. 2007; 26(6):935-942.

Swanson E, Mahgoub A, MacDonald R, Shaukat A. Medical and Endoscopic Therapies for Angiodysplasia and Gastric Antral Vascular Ectasia: A Systematic Review. Clin Gastroenterol Hepatol. 2014; 12(4):571-582.

Ediciones Universidad de Salamanca / @@ఠ $\quad$ FarmaJournal, vol. 4, núm. 2 (2019), pp. 61-72 